\title{
APRENDIZAGEM DE MÁQUINA E APRENDIZAGEM PROFUNDA PARA CLASSIFICAÇÃO DE FALHAS EM EQUIPAMENTOS ROTATIVOS: UMA REVISÃO SISTEMÁTICA
}

Roberto Macedo de Souza1; Herman Augusto Lepikson²; Erick Giovani Sperandio Nascimento ${ }^{3}$; Ubatan Miranda ${ }^{4}$

1, 2, 3, 4 Centro Universitário SENAI CIMATEC, № 1845, Av. Orlando Gomes, - Piatã, 41650-010; Salvador/Ba;

1 rmsouza_2@yahoo.com.br

Resumo: $O$ uso de aprendizagem de máquina e aprendizagem profunda na classificação de falhas em equipamentos rotativos vem sendo ampliado em função da possibilidade de desenvolvimento de modelos computacionais que possam realizar a classificação da falha automaticamente, sem intervenção humana, em estágio inicial, e possibilitar a otimização dos custos de manutenção. O objetivo deste trabalho é realizar uma revisão sistemática em trabalhos científicos publicados entre o período de 2016 a 2019, para identificar o estado da arte. Os resultados indicaram o uso de aprendizagem profunda juntamente com o modelo de Rede Neural Convolucional como o que apresenta maior índice de exatidão e menor trabalho para geração do modelo.

Palavras-Chave: Aprendizagem de Máquina; Aprendizagem profunda; Rede Neural Convulacional; Vibração Mecânica

\section{A SYSTEMATIC REVIEW OF DEEP LEARNING AND MACHINE LEARNING FOR CLASSIFICATION OF FAILURES IN ROTATING EQUIPMENTS}

\begin{abstract}
The use of machine learning and deep learning in the classification of failures in rotating equipment has been expanded due to the possibility of developing computer models that can perform the classification of failure automatically, without human intervention, at an early stage, and enable the optimization of maintenance costs. The objective of this work is to carry out a systematic research in scientific papers published between 2016 and 2019, to identify the state of the art. The results indicated the use of deep learning together with the Convolutional Neural Networks method as the one with the highest accuracy index. However, more in-depth studies on this technique are still needed in order to classify failures.
\end{abstract}

Keywords: Machine Learning, Deep Learning, Convulational Neural Networks; Mechanical Vibration. 


\section{INTRODUÇÃO}

Máquinas rotativas são equipamentos amplamente empregados na indústria. O seu uso contínuo gera desgaste das peças e, portanto, necessita de um monitoramento contínuo para evitar falhas prematuras e paradas inesperadas e, dessa forma, diminuir os custos de manutenção, além de gerenciar as condições do equipamento para que funcione o máximo de tempo em condições seguras e conforme recomendações do fabricante.

Sob a pressão do custo de mão de obra e demanda do mercado, a implementação de sistemas inteligentes que possam tomar decisões automaticamente tornou-se uma forma promissora de equilibrar os custos financeiros. Consequentemente, a monitoração contínua, eficaz e automática é importante para proteger, revelar início de falhas e reduzir o risco de avarias do equipamento.

Uma das formas de monitorar o estado operacional de um equipamento rotativo é através da análise de vibração mecânica, que permite detectar o início de uma falha, realizar diagnósticos e informar a peça defeituosa.

Com o objetivo de implementar melhorias no ambiente industrial, pesquisas são realizadas utilizando Machine Learning (em português, Aprendizagem de Máquina) e Deep Learning (em português, Aprendizagem Profunda) aplicadas na área de análise de vibração para diagnosticar, detectar e classificar falhas em equipamentos rotativos. Portanto, através do uso de inteligência computacional, está sendo possível desenvolver sistemas capazes de ampliar o tempo de vida útil dos equipamentos.

Nesse contexto, o objetivo deste trabalho é realizar uma revisão sistemática, com foco em identificar técnicas que estão sendo pesquisadas e apresentadas, através de trabalhos científicos, que abordem assuntos relacionados a aprendizagem de máquina, aprendizagem profunda e análise de vibração de equipamentos rotativos, apresentem o estado da arte e, através dos seus resultados, propor um modelo capaz de realizar a classificação de falhas com maior exatidão.

Este trabalho está organizado em cinco seções: a seção 1 apresenta a introdução; seção 2 descreve a metodologia utilizada; seção 3 apresenta os resultados e discussões; seção 4 apresenta as considerações finais e sugestões de trabalhos futuros; seção 5 apresenta as referências.

\section{METODOLOGIA (ARIAL 12)}

Para identificar os trabalhos científicos que pudessem contribuir com este, foi definido um conjunto de palavras chaves, período e critérios de inclusão e exclusão.

Para concepção desse estudo foram utilizadas as bases científicas do Google Acadêmico e ScienceDirect.

A string de pesquisa utilizada foi aprimorada durante o estudo de revisão, sendo dividida em dois momentos: 
No primeiro momento foi utilizada a string de busca:

("Deep Learning” OR “Machine Learning”) AND "Mechanical vibration".

No segundo momento, através da leitura de artigos e verificando uma maior tendência do uso do modelo Convolutional Neural Network (CNN - em português, Rede Neural Convolucional) em apresentar maior exatidão, foi alterada a string de busca para:

\section{"Deep Learning" AND "Mechanical Vibration" AND CNN}

Este conjunto de palavras foi utilizado para identificar os trabalhos acadêmicos que referenciam aprendizagem de máquina ou aprendizagem profunda aplicada na classificação de falha de equipamentos rotativos provocada por vibração mecânica.

Os critérios de inclusão e exclusão foram pré-estabelecidos em todos os trabalhos, refinando os resultados obtidos e selecionando os que se aprofundassem no assunto de interesse.

Considerou-se os trabalhos acadêmicos que foram revisados por pares, artigos publicados em revistas com qualis $\mathrm{A} 1$ ou $\mathrm{A} 2$, publicado nos últimos três anos (2016 2019) e análise do título, resumo e palavra-chave para identificar a significância com o objetivo da pesquisa.

Por fim, as questões de pesquisa foram definidas como:

1 - Quais são os trabalhos acadêmicos que referenciam classificação de falha em equipamentos rotativos provocadas por vibração mecânica e utilizam Inteligência Computacional?

2 - Quais são os modelos empregados que apresentam maior exatidão?

3 - Quais são os modelos que requerem menos trabalhos na sua geração?

Através destes critérios, foram selecionados 48 trabalhos considerados relevantes para esta revisão sistemática, mas nem todos serão retratados neste artigo.

\section{RESULTADOS E DISCUSSÃO (ARIAL 12)}

Os trabalhos identificados como relevantes foram divididos em dois grupos, sendo um grupo utilizando aprendizagem de máquina e outro grupo utilizando aprendizagem profunda.

\subsection{Classificação de falha utilizando aprendizagem de máquina}

Observou-se em Marins et al. [1] que o uso de aprendizagem de máquina utilizando o modelo Similiarity Based Modeling (SBM - em português Modelagem Baseado em Similaridade) pode ser utilizado como detector ou classificador de falha automático. Neste trabalho o modelo SBM foi utilizado como detector e gerou as features para o modelo Random Forest que é responsável pela classificação.

Os dados da simulação de falha de desbalanceamento, desalinhamento (vertical e/ou horizontal) e de rolamento (elementos girantes, pistas internas ou 
externas) foram obtidos através de bases de dados públicas (MaFaulDa [2] e CWRU [3]), sendo que esta última foi utilizada para simular as falhas com rolamentos.

Em relação a base de dados CWRU, os autores concluíram que a precisão está próxima das encontradas em outros trabalhos que utilizaram os classificadores do tipo k-NN (k-Vizinhos mais Próximos), Naive Bayes e SVM (Máquina Vetorial de Suporte) e encontraram exatidão de $98,83 \%, 98 \%$ e $98,97 \%$, respectivamente. A exatidão obtida nos modelos testados foi de $98,5 \%$ e $98,9 \%$, quando utilizadas as bases de dados MaFaulDa e CWRU, respectivamente.

Em Liu et al. [4], o trabalho concentrou nas técnicas de aprendizagem de máquina utilizando alguns modelos de classificação de falhas tais como: $k-N N$, Naive Bayes, SVM, Artificial Neural Network (Rede Neural Artificial). O trabalho apresentou as vantagens, limitações e implicações práticas dos diferentes algoritmos e informa que os modelos baseados em aprendizagem profunda têm sido o estado da arte em uma grande variedade de aplicações.

A tabela 1 apresenta vantagens e limitações de alguns modelos analisados.

Tabela 1. Vantagens e limitações dos algoritmos de Inteligência Computacional

\begin{tabular}{|c|c|c|c|}
\hline $\begin{array}{c}\text { Tipo de } \\
\text { Aprendizagem }\end{array}$ & Modelo & Vantagens & Limitações \\
\hline \multirow{7}{*}{$\begin{array}{l}\text { Aprendizagem } \\
\text { de Máquina }\end{array}$} & k-NN & $\begin{array}{l}\text { Teoria madura e fácil } \\
\text { implementação } \\
\text { Pode ser usada para } \\
\text { classificação e regressão }\end{array}$ & $\begin{array}{l}\text { Alto consumo } \\
\text { computacional } \\
\text { Precisa de um } \\
\text { grande espaço de } \\
\text { disco }\end{array}$ \\
\hline & \multirow[t]{2}{*}{$\begin{array}{l}\text { Naive } \\
\text { Bayes }\end{array}$} & $\begin{array}{l}\text { Robusto para valores } \\
\text { perdidos }\end{array}$ & $\begin{array}{l}\text { Explosão } \\
\text { combinatória e } \\
\text { problema } \\
\text { computacional }\end{array}$ \\
\hline & & $\begin{array}{l}\text { Requer pouco espaço de } \\
\text { disco }\end{array}$ & \\
\hline & \multirow[t]{2}{*}{ SVM } & $\begin{array}{l}\text { Alta exatidão de } \\
\text { classificação }\end{array}$ & $\begin{array}{l}\text { Baixa eficiência para } \\
\text { Big Data }\end{array}$ \\
\hline & & $\begin{array}{l}\text { Pode lidar com features de } \\
\text { alta dimensão }\end{array}$ & \\
\hline & \multirow[t]{2}{*}{ ANN } & $\begin{array}{l}\text { Alta exatidão de } \\
\text { classificação }\end{array}$ & $\begin{array}{l}\text { Muitos parâmetros e } \\
\text { ocorre over-fitting } \\
\text { facilmente }\end{array}$ \\
\hline & & $\begin{array}{l}\text { Boa aproximação para } \\
\text { funções complexas não } \\
\text { lineares }\end{array}$ & $\begin{array}{l}\text { Procedimento de } \\
\text { treinamento não } \\
\text { pode ser visto }\end{array}$ \\
\hline \multirow{2}{*}{$\begin{array}{l}\text { Aprendizagem } \\
\text { profunda }\end{array}$} & & $\begin{array}{l}\text { Não precisa de extrator de } \\
\text { features }\end{array}$ & $\begin{array}{l}\text { Precisa de grande } \\
\text { quantidade de } \\
\text { amostras }\end{array}$ \\
\hline & & $\begin{array}{l}\text { Recursos de aprendizagem } \\
\text { e reconhecimento } \\
\text { automático de falhas }\end{array}$ & $\begin{array}{l}\text { Fase de treino } \\
\text { demora muito tempo }\end{array}$ \\
\hline
\end{tabular}




\subsection{Classificação de falha utilizando aprendizagem profunda}

A partir das revisões de trabalhos referenciados abaixo, verificou-se que diversas aplicações para classificação de falha de equipamentos rotativos utilizando sinais de vibração apresentam maior exatidão quando utilizam técnicas de aprendizagem profunda, utilizando o modelo CNN. Portanto, conforme citado no item 2, alteramos os descritores para "Deep Learning" AND "Mechanical Vibration" AND CNN para identificar trabalhos que apresentem melhor exatidão.

O trabalho apresentado por Li, Wang e Wang [5] emprega aprendizagem profunda e os modelos Back Propagation Neural Network (Rede Neural de Propagação de Retorno), Deep Belief Network (Rede de Convicção Profunda), SVM, K-Nearest Neighbours Classification (Classificação dos k-Vizinhos mais Próximos), e Deep Neural Network (Rede Neural Profunda), para realizar a classificação de falha de equipamentos rotativos.

O trabalho apresentou a exatidão encontrada nos modelos BPNN, DBN, SVM, KNNC e DNN de 99,77\%, 99,8\%, 99,85\%, 99,85\% e 99,87\%, respectivamente.

Conforme Zhao et al. [7], o uso do sinal bruto em aplicações utilizando aprendizagem profunda é uma nova área de diagnóstico de falhas de motores de indução orientada por dados, pois facilita muito o trabalho na fase de desenvolvimento do modelo e não requer muito trabalho por parte do desenvolvedor da solução. A grande vantagem de poder utilizar os dados brutos do sinal de vibração é que não necessita da conversão destes em características vetoriais e estatísticas, tais como desvio padrão, curtose e médias para então poder gerar o modelo. A geração do modelo utilizando os dados brutos é mais fácil de ser implementada.

Foi apresentado neste trabalho uma visão geral sobre alguns modelos baseados em aprendizagem profunda em sistemas de Monitoramento de Saúde de Equipamentos e seu impacto nas tecnologias de ponta (estado da arte). O trabalho realizou uma comparação entre alguns modelos de aprendizagem profunda (Random Forest, Neural Network, Autoencoder, Denoising Autoencoder e CNN) e apresentou os erros de cada modelo, demonstrando que, para os modelos apresentados, CNN obteve o menor erro médio absoluto.

Os modelos e respectivos erros médios são apresentados na tabela 2.

O trabalho conclui que os modelos de aprendizagem profunda são capazes de alcançar desempenhos superiores no campo do monitoramento do estado da máquina em comparação aos modelos desenvolvidos utilizando aprendizagem de máquina.

O modelo Deep Convolutional Neural Networks with First-layer Kernels (Redes Neurais Convolucionais Profundas com Kernels de Primeira Camada), foi apresentado por Zhang et al. [8], obteve uma exatidão de $100 \%$ no diagnóstico de falha de rolamentos utilizando uma base de dados pública denominada CWRU. 
Tabela 2. Comparação dos erros dos modos de aprendizagem profunda

\begin{tabular}{|l|r|r|}
\hline Modelo & \multicolumn{1}{c}{$\begin{array}{c}\text { Erro médio } \\
\text { absoluto }\end{array}$} & $\begin{array}{r}\text { Erro médio } \\
\text { quadrático }\end{array}$ \\
\hline Linear SVR & 13,7 & 248,9 \\
\hline Random Forest & $14: 4 \pm 0: 2$ & $289 \pm 5: 9$ \\
\hline Neural Network & $11: 5 \pm 1: 2$ & $191: 6 \pm 26: 8$ \\
\hline Auto-encoder & $11: 2 \pm 1: 9$ & $185 \pm 41: 9$ \\
\hline DBN & $11: 7 \pm 1: 3$ & $194: 6 \pm 34: 2$ \\
\hline CNN & $11: 0 \pm 1: 3$ & $197: 2 \pm 30: 9$ \\
\hline
\end{tabular}

Em Janssens et al. [9] propõe um estudo que consiste no desenvolvimento de uma solução de aprendizagem profunda, que pode aprender, sem intervenção humana, as características dos dados brutos que representam falha de máquina. Durante os experimentos foram analisados rolamentos com falha, rolamentos bons e rotor com desbalanceamento. Ao final do trabalho, dois sistemas são comparados, utilizando os mesmos dados, e observa-se que a precisão obtida pelo sistema desenvolvido pelo autor, utilizando feature-learning e CNN, em detrimento de outro sistema que utiliza feature-learning e o modelo Random Forest, atingiu a precisão de $93,61 \%$ e $87,25 \%$, respectivamente.

No trabalho de Guo, Chen e Shen [10] propõe o uso de um modelo que os autores denominaram de Adaptativo-CNN para diagnosticar falhas em rolamentos e determinar sua gravidade, utilizando a base de dados CWRU. O trabalho obteve uma exatidão próxima a 100\% em classificação de falhas e severidade.

Neste trabalho foi utilizado $50 \%$ da base de dados para teste, os demais dados foram utilizados para treinamento e a análise de falha se limitou a rolamento. Portanto, existe espaço para outras pesquisas em análise de falha provocado, por exemplo, desalinhamento, desbalanceamento e peças soltas.

\section{CONCLUSÃO}

Este artigo teve como objetivo identificar trabalhos acadêmicos relevantes e atualizados que descrevem sobre o uso de aprendizagem de máquina ou aprendizagem profunda para classificação de falhas provocada por vibração mecânica em equipamentos rotativos.

Após leituras e análises dos trabalhos referenciados, observou-se um número crescente de aplicações de classificação de falhas em equipamentos rotativos utilizando aprendizagem profunda com exatidão próxima a 100\%, mesmo que se aplicando a enfoques específicos. Dentre os modelos que estão sendo desenvolvidos, CNN está apresentando maior exatidão, é capaz de superar os modelos tradicionais nesta área e não requerem muito trabalho na geração do modelo.

Observou-se uma lacuna e a necessidade de pesquisa em trabalhos referenciando modelos que foram gerados através de dados extraídos de equipamentos em condições industriais. 
Como futuros passos, esta revisão será estendida a mais bases científicas como IEEE Explore e Scopus objetivando identificar outros modelos que possam apresentar uma exatidão similar ou superior à exatidão apresentada pelo modelo CNN.

\section{REFERÊNCIAS}

[1] MARINS, M. A. et al. Improved Similarity-Based Modeling for the classification of rotating-machine failures. Journal of the Franklin Institute, v. 355, n. 4, p. 19131930, mar. 2018. Disponível em:

https://www.sciencedirect.com/science/article/pii/S0016003217303678. Acesso em: 15 abr. 2019.

[2] UNIVERSIDADE FEDERAL DO RIO DE JANEIRO. Machinery Fault Database. MaFaulDa. Disponível em: http://www02.smt.ufrj.br/ offshore/. Acesso em 26 jun. 2018

[3] CASE WESTERN RESERVE UNIVERSITY - CWRU. Disponível em: https://csegroups.case.edu/bearingdatacenter/pages/download-data-file. Acesso em: 05 Abril 2019.

[4] LIU, R. et al. Artificial intelligence for fault diagnosis of rotating machinery: A review. Mechanical System and Signal Processing, v. 108, p. 33-47, ago. 2018. Disponivel em:

https://www.sciencedirect.com/science/article/pii/S0888327018300748. Acesso em 14 abr. 2019

[5] LI, Z; WANG, Y.; WANG, K. A deep learning driven method for fault classification and degradation assessment in mechanical equipment. Computers in industry, v. 104, p. 1-10, jan. 2019. Disponível em:

https://www.sciencedirect.com/science/article/pii/S0166361517307832. Acesso em 15 abr. 2019

[6] CHATTOPADHYAY, P. et al. Deep learning in fault diagnosis of induction motor drives. Prognostics and System Health Management Conference (PHMChongqing), Chongqing, p. 1068-1073, out. 2018. Disponível em: https://ieeexplore.ieee.org/abstract/document/8603497/authors\#authors. Acesso em 14 mai. 2019

[7] ZHAO, R et al. Deep learning and its applications to machine health monitoring. Mechanical System and Signal Processing, v. 115, p 213-237, jan. 2019. Disponível em https://www.sciencedirect.com/science/article/pii/S0888327018303108. Acesso em 28 jul. 2019

[8] ZHANG, W. et al. A new Deep Learning model for fault diagnosis with good antinoise and domain adaptation ability on raw vibration signals. Sensors, v. 17, fev. 2017. Disponível em https://www.mdpi.com/1424-8220/17/2/425. Acesso em 28 jul. 2019

[9] JANSSENS, O. et al. Convolutional Neural Network based fault detection for rotating machinery, Journal of Sound and Vibration, v. 377, p 331-345, set. 
2016. Disponível em https://www.sciencedirect.com/science/article/pii/S0022460X16301638. Acesso em 20 jul. 2019

[10] GUO, X.; CHEN, L.; SHEN, C. Hierarchical adaptive deep convolution neural network and its application to bearing fault diagnosis, Measurement, v. 93, $p$ 490-502, nov. 2016. Disponível em https://www.sciencedirect.com/science/article/pii/S0263224116304249. Acesso em 28 jul. 2019 